Radiologe 2020 60 :475-476

https://doi.org/10.1007/s00117-020-00698-y

(c) Springer Medizin Verlag $\mathrm{GmbH}$, ein Teil von Springer Nature 2020

\section{M.-A. Weber' $\cdot$ T. Mittlmeier ${ }^{2}$}

${ }^{1}$ Institut für Diagnostische und Interventionelle Radiologie, Kinder- und Neuroradiologie, Universitätsmedizin Rostock, Rostock, Deutschland

${ }^{2}$ Klinik und Poliklinik für Chirurgie, Abteilung für Unfall-, Hand- und Wiederherstellungschirurgie, Universitätsmedizin Rostock, Rostock, Deutschland

\title{
Bildgebende Frakturdiagnostik und Frakturklassifikationen - Teil 1
}

Liebe Leserinnen und Leser,

die Diagnostik von Frakturen gehört zum täglichen Brot des Radiologen. Dabei hat sich der enge Austausch mit den Traumatologen bewährt - „4 Augen sehen mehr als 2 Augen", und Unfallmechanismus und Schmerzsymptomatik sind wichtige klinische Bausteine bei der Interpretation der bisweilen subtilen Frakturzeichen im Röntgen. Daher hilft es dem Radiologen ungemein, wenn diese Informationen in den klinischen Angaben genannt werden und sich nicht nur auf das bisweilen praktizierte „Ausschluss \#“ beschränken. Ebenfalls sollte man nicht dem „satisfaction of search error" erliegen, sich also mit einer gefundenen Pathologie zufriedengeben, sondern systematisch das gesamte Röntgenbild bzw. alle vorhandenen MRT-Sequenzen oder CTBilder durchmustern [1]. Auch hier ist die Kenntnis typischer Frakturformen und deren Begleitverletzungen wichtig, um systematisch nach typischen Begleitverletzungen zu suchen. Das Wort Fraktur leitet sich aus dem Lateinischen "frangere", d.h. brechen ab. Neben der uns Radiologen und Traumatologen gut bekannten Definition als Unterbrechung der Kontinuität eines Knochens unter Bildung zweier oder mehrerer Bruchstücke steht Fraktur auch für die Druckschrift mit gebrochenen, d. h. nicht gerundeten Linien. Die Frakturschrift war in gedruckten Dokumenten von Mitte des 16. bis Anfang des 20. Jahrhunderts die meistbenutzte Druckschrift im deutschsprachigen Raum [2]. Und selbst in eine Redewendung hat der Begriff „Fraktur“ Eingang gefunden: Wer mit jemandem "Fraktur redet", redet mit diesem in "geraden, deutlichen oder groben Buchstaben", redet also in klaren, deutlichen Worten, ohne Umschweife oder Euphemismen, und sagt diesem direkt und unverblümt die Meinung. Die Diagnostik und Behandlung von Frakturen begleitet die Menschheitsgeschichte und war naturgemäß über Jahrtausende eine konservative Therapie, bereits im Papyrus Edwin Smith (ca. 1500 v. Chr.) wurde ein Verband zur Ruhigstellung von Gelenkfortsatzfrakturen des Unterkiefers beschrieben, und Hippokrates führte um ca. 400 v. Chr. eine zur Ruhigstellung dienende Ledermanschette ein [3]. Auch ohne Röntgendiagnostik war zwar das Verständnis für die $\mathrm{zu}$ Grunde liegenden Veränderungen und Verletzungen schon zu Zeiten von Hippokrates entwickelt. Zum Einrichten von Frakturen und Luxationen wurden in den folgenden Jahrhunderten verschiedene Techniken und auch Apparate entwickelt, da man erkannt hatte, dass die Funktion des Bewegungsapparates von seiner Form abhängig war. Nur konnte man mit den zur Verfügung stehenden Mitteln eine Ausheilung in der normalen, anatomischen Situation selten erreichen und musste Fehlstellungen und somit auch Funktionsstörungen akzeptieren. Erst Meilensteine der Medizingeschichte wie die Einführung der Anti- und Asepsis, die Entwicklung von Narkosemitteln und vor allem auch die Röntgendiagnostik 1895 versetzten Ärz- te im ausgehenden 19. und beginnenden 20. Jahrhundert zunehmend in die Lage, gezielt Frakturen zu behandeln. Im Jahr 1959 nahm mit dem Zusammenschluss der Arbeitsgemeinschaft für Osteosynthesefragen (AO) der Fortschritt in der operativen Knochenbruchbehandlung stark an Geschwindigkeit auf unter der Doktrin der anatomischen Reposition und stabilen Fixation aller Fragmente [4].

Genug zur Historie: In Der Radiologe haben wir für Sie zwei Themenhefte zu der bildgebenden Frakturdiagnostik und den Frakturklassifikationen gestaltet, die diverse Aspekte der Frakturen einerseits und Besonderheiten der Frakturen an verschiedenen Skelettabschnitten andererseits beleuchten. Eingeführt wird das erste Themenheft mit dem Beitrag von C. Fischer zur Allgemeinen Frakturlehre und ihrem bildgebenden Korrelat; auch Pseudarthrosen werden ausführlich behandelt. Es folgen Beiträge zu den speziellen Aspekten von Frakturen bei Kindern und Jugendlichen (C. Hauenstein et al.), von den Besonderheiten von pathologischen Frakturen (F. Wünnemann et al.) und von Stressfrakturen (K. Thierfelder et al.). Von den Unfallchirurgen (A. Sckell und T. Mittlmeier) werden des Weiteren Implantate für die Frakturversorgung vorgestellt, die wir Radiologen bei der Befundung kennen sollten. Zwei Beiträge zu den Besonderheiten und Klassifikationen der Frakturen an der unteren Extremität folgen: J. Berger-Groch et al. zu proximalen Femur- und Tibiafrakturen und G. Bratke et al. zum dis- 
talen Unterschenkel, Sprunggelenk und Fuß. Abgerundet wird das Themenheft von einem Beitrag mit Fokus auf die Frakturdiagnostik von Schulter und Schultergürtel (C. von Falck und N. Hawi).

Wir hoffen Ihnen, liebe Leserinnen und Leser, mit diesem ersten der beiden Themenhefte eine gute Zusammenfassung aktueller Themenfelder der bildgebenden Frakturdiagnostik und einen Überblick über die gängigen Frakturklassifikationen zu präsentieren. Die nach klinischen Belangen 2018 komplett überarbeitete AO-Klassifikation inkludiert die neu entwickelte Klassifikation der pädiatrischen Frakturen der langen Röhrenknochen und bietet uns die Möglichkeit, eine gemeinsame Sprache bei der Frakturdeskription zu finden [5], die Literaturquelle ist im Internet frei zugänglich.

Wir bedanken uns auf das Allerherzlichste bei den Autoren für deren hervorragende Beiträge, wünschen Ihnen viel Spaß beim Lesen und würden uns sehr freuen, wenn Sie diese Zusammenstellung interessant und hilfreich für Ihre tägliche praktische Arbeit finden.

Mit den besten Grüßen

Prof. Dr. Marc-André Weber

Prof. Dr. Thomas Mittlmeier

\section{Korrespondenzadresse}

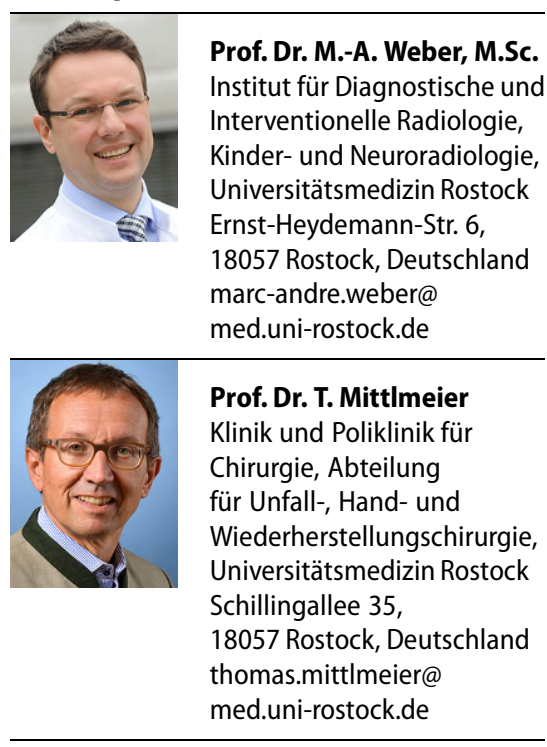

Interessenkonflikt. M.-A. Weber und T. Mittlmeier geben an, dass kein Interessenkonflikt besteht.

\section{Literatur}

1. Kim YW, Mansfield LT (2014) Fool me twice: delayed diagnoses in radiology with emphasis on perpetuated errors. AJR Am J Roentgenol 202:465-470

2. Typolexikon (2019) Fraktur. https://www. typolexikon.de/fraktur-schrift/. Zugegriffen: 25. Apr. 2020

3. Eulert S (2002) Die Behandlung von Gelenkfortsatzfrakturen des Unterkiefers unter besonderer Berücksichtigung der Würzburger ZugschraubenPlatte. https://opus.bibliothek.uni-wuerzburg. de/opus4-wuerzburg/frontdoor/deliver/index/ docld/246/file/s_eulert.pdf. Zugegriffen: 25. Apr. 2020

4. Heim UF (2012) Geschichte der Entstehung und erste Jahre der Arbeitsgemeinschaft für Osteosynthesefragen 1952-1963. OP-JOURNAL 28:98-106

5. Meinberg E, Agel J, Roberts C, Karam MD, Kellam JF (2018) Fracture and dislocation classification compendium-2018.J Orthop Trauma 32(1):S1-S170
Möchten Sie einen Beitrag für Der Radiologe einreichen?

Wir freuen uns, dass Sie unsere Zeitschrift Der Radiologe mitgestalten möchten.

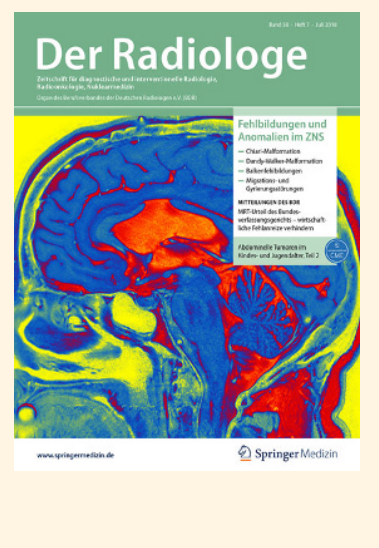

Für folgende Rubriken können Manuskripte eingereicht werden:

- Übersichten

- Originalien

- Quiz/Wie lautet Ihre Diagnose?

Um Ihnen bei der Manuskripterstellung behilflich zu sein, haben wir für unsere Autoren ausführliche Autorenleitfäden und Musterbeiträge für die verschiedenen Rubriken zusammengestellt.

Diese und weitere Hinweise zur Manuskripterstellung finden Sie online unter dem Menüpunkt „Hinweise für Autoren“ unter www.DerRadiologe.de.

Bitte reichen Sie Ihren fertigen Beitrag in elektronischer Form bei den zuständigen Schriftleitern ein:

\section{Übersichten/Originalien:}

Prof. Dr. Christian Herold, Wien

ute.weber-woisetschlaeger@

meduniwien.ac.at

Quiz

Prof. Dr. Thomas Helmberger, München thomas.helmberger@

klinikum-muenchen.de

Sollten Sie noch Fragen zur Manuskriptgestaltung und zur Verwendung von DICOMMaterial haben, wenden Sie sich bitte an die Redaktion:

Claudia Zappe

claudia.zappe@springernature.com

Wir freuen uns auf Ihre Beitrage! 